\title{
Cluster Preface: Organosulfur and Organoselenium Compounds in Catalysis
}

Ying-Yeung Yeung

Department of Chemistry, The Chinese University of Hong Kong, Shatin, NT, Hong Kong, P. R. of China yyyeung@cuhk.edu.hk

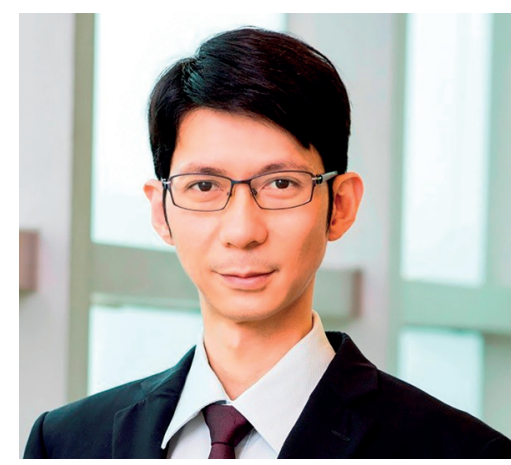

Received: 01.08.2019

Accepted: 01.08.2019

Published online:

DOI: 10.1055/s-0039-1690021; Art ID: st-2019-w5000-c

Ying-Yeung Yeung received his B.Sc. (2001) at The Chinese University of Hong Kong. He continued his graduate research at the same university under the supervision of Prof. Tony K. M. Shing. After four years (2001-2005) of research dedicated toward natural product synthesis, he moved to the USA to conduct postdoctoral research with Prof. E. J. Corey at Harvard University (2005-2008). In 2008, he joined the National University of Singapore, Department of Chemistry. In 2015, he moved to The Chinese University of Hong Kong as an associate professor. He has been the department chairman (since 2016) and a full professor (since 2019). His research interests include asymmetric catalysis, green oxidation, and methodology development.

Sulfur and selenium are group 16 elements (chalcogens). While organosulfur and organoselenium compounds have been recognized as useful reagents in synthetic organic chemistry since the 1970s, the use of organosulfur/selenium in catalysis has emerged in recent years. Catalysis plays a central role in modern synthetic chemistry. Thus, significant efforts have been devoted to the development of various catalytic protocols in the past decades. For catalyst structure design, oxygen, nitrogen and phosphorus are employed as the constitutional elements in many circumstances. For example, amines are frequently used as Lewis bases in organocatalysis and as ligands in transition-metal catalysis. Chalcogenides, including sulfides and selenides, are a separate class of Lewis base. The relatively soft Lewis basic nature of organosulfur and organoselenium compounds leads to interesting catalytic performance as compared with hard Lewis bases such as amines. As a result, research efforts to explore the chemistry of organosulfur and organoselenium compounds as organocatalysts and ligands for transition metals in various catalytic processes have received considerable attention from the scientific community.
Unlike oxygen, which is also a group 16 element, sulfur and selenium are multivalent in nature. As a result, they have fruitful chemistry in catalytic oxidation reactions. Other than applications as Lewis bases, using organochalcogens as soft Lewis acids through chalcogen bond interaction has been studied in biological systems and in crystal engineering. This interaction has emerged as a new tool in catalytic organic reactions in the past few years.

Despite the usefulness of organosulfur/selenium compounds as catalysts, the odor of sulfur and selenium reagents and inefficient classical preparation procedures are obstructions in this research field. As a result, the development of more efficient and preferentially odorless methodologies to introduce $\mathrm{C}-\mathrm{S}$ and $\mathrm{C}-\mathrm{Se}$ bonds is highly relevant to boosting the progress of organochalcogen catalysis.

In this SYNLETT Cluster, we are delighted to feature 11 important contributions dedicated toward different themes and concepts related to organosulfur/selenium catalysis. These endeavors include the development of new reactions and efficient methods for the preparation of chalcogen compounds, as well as a mechanistic study.

Lewis basic chalcogen-catalyzed electrophilic functionalizations of olefinic substrates are very useful reactions which allow the rapid construction of structurally complex cyclic molecules in a single chemical operation. Halogen and chalcogen electrophilic reagents have been widely utilized. The resulting cyclized products are valuable building blocks of biologically relevant molecules. In addition, the halogen/chalcogen functionalities can easily be manipulated to give various derivatives. Some new catalytic protocols for electrophilic cyclizations are reported in this Cluster, including catalyst-controlled regio- and stereoselective bromolactonization of stilbene-type carboxylic acids with chiral bifunctional sulfides (S. Shirakawa) ${ }^{1}$ and enantioselective halo-/selenocyclization of olefinic acids with $C_{2}$ symmetric sulfur-based catalysts, which gave rise to 
a range of normal- and medium-sized cyclic lactones (S. Kumar). ${ }^{2}$ A detailed study of the kinetic profiles for polyene sulfenocyclizations catalyzed by Lewis basic selenides has provided very useful information on the mechanistic aspects of such transformations (S. E. Denmark). ${ }^{3}$

Organoselenium compounds are stable at various oxidation states and have been utilized as catalysts in several oxidation reactions. Benzeneseleninic acid, which is generated in situ from diphenyl diselenide, was found to be effective in catalyzing the oxidative degradation of benzoins into benzoic acids with hydrogen peroxide as the stoichiometric oxidant (H. Cao and X. Zhang). ${ }^{4}$ Diphenyl diselenide could also be oxidized by NFSI to generate phenylselenyl fluoride in situ, which effectively catalyzed aza-Wacker reactions of olefinic hydrazones and oximes to give isoquinolinium imides and an isoquinoline $\mathrm{N}$-oxide, respectively (X. Zhao). ${ }^{5}$ Other than small molecules, the synthesis of polyanilines through the organoselenium-catalyzed oxidation of anilines is reported $\left(L\right.$. Yu and Q. Xu) ${ }^{6}$

Compared to Lewis base catalysis, using organochalcogens as acid catalysts ( $\pi$-acids and $\sigma$-hole catalysts) are less studied. Nonetheless, the use of chiral organoselenium compounds as $\pi$-acid catalysts for enantioselective iminolactonization is reported (K. Maruoka and T. Hashimoto). ${ }^{7}$ The use of sulfur- and selenium-based bidentate cationic chalcogen bond donors to catalyze the reduction of quinolines (through $\sigma$-hole interactions) is also described (S. M. Huber $)^{8}$

Other than being organocatalysts, organosulfur compounds can serve as ligands for transition-metal catalysis. A report on using chiral-sulfonamide-based olefin ligands for the rhodium-catalyzed enantioselective addition of glyoxylate with arylboronic acids is reported (M.-H. Xu) ${ }^{9}$

Some efficient methods to introduce sulfur and selenium to build catalyst scaffolds are also presented in this Cluster. Dialkyl diselenides are useful catalysts for various oxidation reactions. However, classical preparation methods often rely on malodorous starting materials such as selenols. The odorless reagent sodium selenosulfate, which can readily be synthesized from sodium sulfite and elemental selenium, has been utilized in the synthesis of various dialkyl diselenides ( $L$. Yu and X. Jiang). ${ }^{10}$ The formation of C$S$ bonds typically requires harsh conditions and an elevated temperature. Alternatively, photocatalytic electrophilic benzothialation to give benzothiazoles and direct $\mathrm{C}-\mathrm{H}$ sulfenylation of indoles are reported (J. L. Gustafson). ${ }^{11}$

The important contributions clearly showcase that the research field of organosulfur and organoselenium catalysis is dynamic and ever-growing. I am very grateful to all the distinguished scientists who have contributed to this SYNLETT Cluster.

\section{Y.-Y. Yeung, \\ Hong Kong, June 2019}

\section{References}

(1) Tsuchihashi, A.; Shirakawa, S. Synlett 2019, 30, 1662.

(2) Jana, S.; Verma, A.; Rathore, V.; Kumar, S. Synlett 2019, 30, 1667.

(3) Robb, K. A.; Athavale, S. V.; Denmark, S. E. Synlett 2019, 30, 1656.

(4) Cao, H.; Chen, T.; Yang, C.; Ye, J.; Zhang, X. Synlett 2019, 30, 1683.

(5) Li, H.; Liao, L.; Zhao, X. Synlett 2019, 30, 1688.

(6) Gao, G.; Han, J.; Yu, L.; Xu, Q. Synlett 2019, 30, 1703.

(7) Otsuka, Y.; Shimazaki, Y.; Nagaoka, H.; Maruoka, K.; Hashimoto, T. Synlett 2019, 30, 1679.

(8) Wonner, P.; Steinke, T.; Huber, S. M. Synlett 2019, 30, 1673.

(9) Chen, D.; Liu, J.-G.; Zhang, X.; Xu, M.-H. Synlett 2019, 30, 1693.

(10) Liu, Y.; Ling, H.; Chen, C.; Xu, Q.; Yu, L.; Jiang, X. Synlett 2019, 30 , 1698.

(11) Dinh, A. N.; Nguyen, A. D.; Aceves, E. M.; Albright, S. T.; Cedano, M. R.; Smith, D. K.; Gustafson, J. L. Synlett 2019, 30, 1648. 